\title{
Is megasequencing madness?
}

\section{While there is a strong case for sequencing the entire human genome it should not be at the expense of other research. And sociological factors might yet impede the project.}

Human biology has entered a phase where no aspect is, or need be, left untouched by the techniques of molecular genetics. DNA sequencing is set to become a stock-in-trade for all investigators of human physiology or pathology. So the notion has arisen that the study of human biology would benefit enormously were the complete DNA sequence of the human genome to be available. With $3 \times 10^{9}$ base pairs to sequence, the task is formidable. But it is not impossible, given on a conservative estimate, 300 people, 20 years and a thousand million dollars. The question is whether the effort is worth the consumption of such resources. After a period of initial enthusiasm, many molecular biologists have backed off supporting the project in its entirety but not so Professor Walter Gilbert of Harvard University. Speaking at Nature's conference on Exploring the Human Genome, held in Boston last week, Gilbert argued against half measures or delay.

Gilbert points out that the number of base pairs that an individual could be expected to sequence in a year need only increase by 1 or 2 orders of magnitude to between $10^{6}$ and $10^{7}$ for 150 scientists (with 150 support staff) to sequence the entire genome in the course of 20 years. In fact, of course, machines would replace people for much of this work. Indeed, the first automated DNA sequencer will soon be available, and able to handle $10^{6}$ bases a year, according to Leroy Hood, in whose Caltech laboratory the prototype was built.

In the course of the conference Hood voiced a frequently heard view that it would be wiser to spend a few years on developing the technology of automated DNA sequencing and the data processing systems that will be needed to handle the emerging information than to dive right into "megasequencing". To which Gilbert reasonably countered that the project of sequencing the entire human genome would serve to drive the development of the technology, so should be undertaken from the start. In time scale, the outcome of the two approaches is unlikely to differ since even Gilbert estimates that only 1 per cent of the sequence would emerge from the efforts of the first third of the project, with the last third accounting for 90 per cent of the sequence.

Acknowledging that all would not be plain sailing, Gilbert nonetheless made light of problems that, for others, have seemed a serious barrier to completing the sequence. First, there is the problem that arises from the presence in the human genome of a considerable number of sequences that are repeated, sometimes in very many copies and sometimes in tandem. These could create confusion, if not havoc, in attempts to fit together randomly sequenced fragments of the genome. But, says Gilbert, they would present little trouble if the fragments are not random but of known physical relationship to one another, as he proposes. As to the problem of those few sequences that are bound to defy being cloned, for reasons that are not apparent, Gilbert takes the simple view that it does not matter if the "complete" sequence is slightly less than complete. More of a problem, perhaps, is the accuracy of sequencing; one error in a proteincoding sequence can make nonsense out of sense. For manual sequencing, errors are usually the result of carelessness and it will take much development before machines are even as good as careless humans. Ten years ago sequencers made about one mistake in $10^{4}$ bases. Now, said Gilbert, it is more like one in $10^{6}$, a figure he was unable to sustain on questioning.

Set completely apart from these technical snags, whatever their size, is the problem of resources. In that respect, opponents of the complete project have some powerful arguments. One is that it would soak up all the most appropriate technologists, which is likely to be true but not a problem if there is widespread committment to the project. Another is that the biological science community cannot afford to have diverted from other research the sum of $\$ 30$ million a year. Gilbert deflects the problem by saying that the project is not one of research but of the creation of a research tool. It should be considered along the lines of an industrial project both in scale and in the emphasis on quality control. The implicit message is that the project should not vie with genuine research for financial support but should be separately financed. There is no doubt that such a solution would be welcome, and with both the US Department of Energy and the Howard Hughes Institute already involved in discussions, the prospects of finding additional funds seem fair.

Particularly if financial support is to come from such sources, it is probable that it will go to a newly-created centre rather than being dispersed among several existing institutes. Gilbert argues for the centralization of the project in terms of the economy of scale and the efficiency of the operation and if the idea of the project in its entirey is to be embraced, the case for a single centre is powerful.

But should the entire project be embraced? The alternative, which is gaining support, is that it should be much less ambitious, going only as far as the reduction of the genome into a collection of defined fragments of known physical relationship to each other and stopping short of sequencing them. Instead, the fragments of cloned DNA would be freely available to anyone interested in sequencing them for their own specific purposes. In that way, it is argued, the cost and scale of the operation would be contained and yet the outcome would be an invaluable starting point for an eventual sequence of the entire genome.

This has its attractions as a counter proposal. Chief among them is that the sequencing would be carried out with forethought and some expectations, rather than blindly. For example, particular fragments would be sequenced with the firm expectation, based on genetic evidence, that they contained genes that are responsible for heritable diseases. In that way, also, priorities for sequencing would automatically emerge so that the most important parts of the genome, at least by the self-serving definition that they are the parts that are currently receiving attention, would be sequenced first.

The inevitable price to be paid for such an approach is that each laboratory will need its own sequencing team and machinery, and will have to spend time in training technical staff. By the same token, time will be wasted in that competing laboratories will frequently sequence the same fragments. But at that point the problem becomes one of sociology. Can the major molecular biology laboratories agree to a joint effort in sequencing with, perhaps, little say in the operation? Can they give up their psychological addiction to sequencing? Gilbert says that the central purpose of a project designed to sequence the entire human genome is to provide a reference sequence against which variation can be measured by individual laboratories (and in that sense it matters not a jot whose genome is chosen). If somebody will pay for the project it has much to recommend it. But even if the money is found, a host of sociological factors may yet impede biology's entry into "big science".
Peter Newmark 